\title{
Homotopy Characterization of ANR Function Spaces
}

\author{
Jaka Smrekar \\ Fakulteta za Matematiko in Fiziko, Jadranska Ulica 19, 1111 Ljubljana, Slovenia \\ Correspondence should be addressed to Jaka Smrekar; jaka.smrekar@fmf.uni-lj.si
}

Received 5 May 2013; Accepted 25 August 2013

Academic Editor: Yongsheng S. Han

Copyright (c) 2013 Jaka Smrekar. This is an open access article distributed under the Creative Commons Attribution License, which permits unrestricted use, distribution, and reproduction in any medium, provided the original work is properly cited.

Let $Y$ be an absolute neighbourhood retract (ANR) for the class of metric spaces and let $X$ be a topological space. Let $Y^{X}$ denote the space of continuous maps from $X$ to $Y$ equipped with the compact open topology. We show that if $X$ is a compactly generated Tychonoff space and $Y$ is not discrete, then $Y^{X}$ is an ANR for metric spaces if and only if $X$ is hemicompact and $Y^{X}$ has the homotopy type of a CW complex.

\section{Introduction}

Let $Y$ be an absolute neighbourhood retract for metric spaces (henceforth abbreviated as "ANR"). This means that whenever $Y$ is embedded in a metric space as a closed subspace $\widehat{Y}$, there exists a retraction of an open neighbourhood onto $\widehat{Y}$. We refer the reader to the first part of Mardešić [1] for a scenic survey of the theory of ANRs.

Let $X$ be a topological space. The question that this paper is concerned with is when is $Y^{X}$, the space of continuous functions $X \rightarrow Y$, equipped with the compact open topology, also an ANR. A basic result of Kuratowski (see [2, page 284]), which is a consequence of the classical homotopy extension theorem of Borsuk, states that $Y^{X}$ is an ANR if $X$ is a metrizable compactum.

For a negative example, consider the discrete space of natural numbers $\mathbb{N}$ and the two-point discrete $\operatorname{ANR}\{0,1\}$. Then $\{0,1\}^{\mathbb{N}}$ is a Cantor set and hence certainly not an ANR. In fact, as the path components of $\{0,1\}^{\mathbb{N}}$ are not open, it does not even have the homotopy type of a CW complex; that is, it is not homotopy equivalent to any CW complex. As every ANR has the homotopy type of a CW complex, this provides a necessary condition for $Y^{X}$ to be an ANR.

In fact, a topological space has the homotopy type of an ANR if and only if it has the homotopy type of a CW complex (see Milnor [3, Theorem 2]). However, there are numerous examples of spaces that have CW homotopy type but are not ANRs. For example, let $F$ be the topological cone over the convergent sequence $\{0,1,1 / 2,1 / 3, \ldots\}$. Then $F$ is contractible; that is, it has the homotopy type of a one-point CW complex. But as $F$ is not locally path connected, it is not an ANR.

On the other hand, Cauty [4] showed that a metrizable space is an ANR if and only if each open subspace has the homotopy type of a CW complex. It turns out that the space of functions into an ANR inherits a good deal of reasonable behaviour from the target space. Thus, under mild restrictions on $X$, if $Y$ is an ANR and $Y^{X}$ is a metrizable space with the homotopy type of a CW complex, $Y^{X}$ is in fact an ANR. Put another way, $Y^{X}$ is an ANR if and only if $Y$ is an ANR and $Y^{X}$ is a metrizable space with the homotopy type of an ANR.

Basic Definitions and Conventions. A topological space $X$ is called hemicompact if $X$ is the union of countably many of its compact subsets $\left\{K_{i} \mid i\right\}$ which dominate all compact subsets in $X$. This means that for each compact $C \subset X$ there exists $i$ with $C \subset K_{i}$. (The perhaps somewhat noninformative word "hemicompact" was introduced by Arens [5] in relation to metrizability of function spaces. See the beginning of Section 2.)

A space $X$ is compactly generated if the compact subspaces determine its topology. That is, a subset $A$ is closed in $X$ if and only if $A \cap C$ is closed in $C$ for each compact subspace $C$. Such spaces are also commonly called $k$-spaces (see, e.g., Willard [6]). We do not require a hemicompact or a compactly generated space to be Hausdorff. 
A space $X$ is Tychonoff if it is both completely regular and Hausdorff. Locally compact Hausdorff spaces and normal Hausdorff spaces are Tychonoff (examples of the latter are all metric spaces and all CW complexes).

The terms map and continuous function will be used synonymously.

A map $f: Y \rightarrow Y^{\prime}$ is a homotopy equivalence if there exists a map $g: Y^{\prime} \rightarrow Y$ (called a homotopy inverse) for which the composites $g \circ f$ and $f \circ g$ are homotopic to their respective identities. In this case, $Y$ and $Y^{\prime}$ are called homotopy equivalent, and we say that $Y^{\prime}$ has the homotopy type of $Y$.

The following are our main results.

Theorem 1. Let $X$ be a compactly generated hemicompact space and let $Y$ be an ANR. Then $Y^{X}$ is an ANR if and only if $Y^{X}$ has the homotopy type of an $A N R$, which is if and only if it has the homotopy type of a CW complex.

We call a (not necessarily Hausdorff) space locally compact if each point is contained in the interior of a compact set. It is well-known that compactly generated spaces are precisely quotient spaces of locally compact spaces. Compactly generated hemicompact spaces seem to be important enough to warrant an analogous characterization. In the appendix, we prove that they arise as nice quotient spaces of $\sigma$-compact locally compact spaces.

Assuming additional separation properties, Theorem 1 can be strengthened as follows.

Corollary 2. Let $X$ be a compactly generated Tychonoff space and let $Y$ be an ANR which contains an arc. Then $Y^{X}$ is an ANR if and only if $X$ is hemicompact and $Y^{X}$ has the homotopy type of a CW complex.

Theorem 1 is a considerable extension of Theorem 1.1 of [7] where the equivalence was proved using a different technique under the more stringent requirement that $X$ be a countable CW complex. Our proof of Theorem 1 leans on Morita's homotopy extension theorem for $P_{0}$-embeddings (see Morita [8]).

Even when $X$ is a countable CW complex, it is highly nontrivial to determine whether or not the function space $Y^{X}$ has the homotopy type of a CW complex. The interested reader is referred to papers $[7,9,10]$ for more on this.

\section{Proof of Theorem 1}

For subsets $A$ of the domain space and $B$ of the target space, we let $G(A, B)$ denote the set of all maps $f$ that map the set $A$ into the set $B$. For topological spaces $X$ and $Y$, the standard subbasis of the compact open topology on $Y^{X}$ is the collection $\mathscr{P}$ of all $G(K, V) \subset Y^{X}$ with $K$ a compact subset of $X$ and $V$ an open subset of $Y$.

To prove Theorem 1, we use the fact that ANRs for metric spaces are precisely the metrizable absolute neighbourhood extensors for metric spaces (abbreviated as "ANE"); see, for example, $\mathrm{Hu}$ [11, Theorem 3.2]. A space $Y$ is an ANE if every continuous function $A \rightarrow Y$, where $A$ is a closed subspace of a metric space, extends continuously over a neighbourhood of $A$.

Note that if $X$ is a hemicompact space with the sequence of "distinguished" compact sets $\left\{K_{i}\right\}$, the map into the countable Cartesian product

$$
Y^{X} \longrightarrow \prod_{i=1}^{\infty} Y^{K_{i}} \text {, defined by } f \longmapsto\left\{\left.f\right|_{K_{i}}\right\}
$$

is an embedding (see also Cauty [12]). Consequently, if $d_{i}$ denotes the supmetric on $Y^{K_{i}}$ induced by a metric on $Y$, then $Y^{X}$ is metrizable by the metric

$$
d(f, g)=\sum_{i=1}^{\infty} \min \left\{\frac{1}{2^{i}}, d_{i}\left(\left.f\right|_{K_{i}},\left.g\right|_{K_{i}}\right)\right\}
$$

(See Arens [5, Theorem 7].) Given the hypotheses of Theorem 1, therefore, we need to show that for every pair $(Z, A)$ with $Z$ metric and $A$ closed in $Z$, every continuous function $f: A \rightarrow Y^{X}$ extends continuously over a neighbourhood of $A$ in $Z$. We need some preliminary results.

First, we state the classical exponential correspondence theorem with minimal hypotheses. Here, a space is regular if points can be separated from closed sets by disjoint open sets.

Proposition 3. Let $X, Y$, and $Z$ be topological spaces. Let $f: Z \rightarrow Y^{X}$ be any function with set-theoretic adjoint $\widehat{f}: Z \times X \rightarrow Y$. If $\widehat{f}$ is continuous, then $f$ is (well-defined and) continuous. For the converse, suppose that $X$ is locally compact. If $f$ is continuous and, in addition, $X$ is regular or $Y$ is regular, then $\widehat{f}$ is continuous. This accounts for a bijection $\left(Y^{X}\right)^{Z} \leftrightarrow Y^{(X \times Z)}$

Proof. The requirement that $X$ be regular is standard. (See, e.g., [13, Corollary 2.100].) We prove that the continuity of $f$ implies that of $\widehat{f}$ if $X$ is locally compact and $Y$ is regular, as it is apparently not so standard.

Suppose that $f$ is continuous and $\widehat{f}\left(z_{0}, x_{0}\right)=: y_{0}$ lies in the open set $V \subset Y$. As $Y$ is regular, there is an open set $W$ with $y_{0} \in W \subset \bar{W} \subset V$. As $X$ is locally compact, $x_{0}$ is contained in the interior of a compact set $C$. Write $\phi=f\left(z_{0}\right)$. Clearly, $K=\phi^{-1}(\bar{W}) \cap C$ is a compact set contained in $\phi^{-1}(V)$. This means that $f\left(z_{0}\right)$ lies in the open set $G(K, V)$. As $f$ is continuous, there is an open neighbourhood $U$ of $z_{0}$ so that $f(U) \subset G(K, V)$. Consequently, $\widehat{f}(U \times K) \subset V$. As $x_{0}$ lies in the interior of $K, \widehat{f}$ is continuous at $\left(z_{0}, x_{0}\right)$.

Definition 4. For any space $Z$, let $\kappa(Z \times X)$ denote the topological space whose underlying set is $Z \times X$ and has its topology determined by the subsets $Z \times C$ (with the Cartesian product topology) where $C$ ranges over the compact subsets of $X$. That is, $F \subset Z \times X$ is closed in $\kappa(Z \times X)$ if and only if $F \cap(Z \times C)$ is closed in $Z \times C$ for each compact subspace $C$ of $X$. The identity $\kappa(Z \times X) \rightarrow Z \times X$, where the latter has the Cartesian product topology, is evidently continuous. 
In the language of Dydak [14], $\kappa(Z \times X)$ has the covariant topology on $Z \times X$ induced by the class of set-theoretic inclusions $Z \times C \hookrightarrow Z \times X$ where $C$ ranges over the compact subsets of $X$ and the $Z \times C$ carry the product topology.

The introduction of the topology $\kappa(Z \times X)$ is motivated by the following lemma.

Lemma 5. Let $Z$ be any topological space, let $Y$ be a regular space, and let $X$ be a compactly generated hemicompact space. Let $f: Z \rightarrow Y^{X}$ be a function with set-theoretic adjoint $\widehat{f}$ : $X \times Z \rightarrow Y$. Then $f$ is continuous if and only if $\widehat{f}: \kappa(Z \times$ $X) \rightarrow Y$ is continuous. This accounts for a bijection $\left(Y^{X}\right)^{Z} \leftrightarrow$ $Y^{\kappa(Z \times X)}$.

Proof. Let $\left\{K_{i}\right\}$ be the sequence of distinguished compacta in $X$ and let $R_{i}: Y^{X} \rightarrow Y^{K_{i}}$ denote the map that to each function $X \rightarrow Y$ assigns its restriction to $K_{i}$. Clearly $R_{i}$ is continuous. If $f: Z \rightarrow Y^{X}$ is continuous, then so is the composite $R_{i} \circ f: Z \rightarrow Y^{K_{i}}$. By Proposition 3, so is its adjoint $\left.\widehat{f}\right|_{Z \times K_{i}}: Z \times K_{i} \rightarrow Y$. As each compact set $C$ is contained in one of the $K_{i}$, it follows by definition of $\kappa(Z \times X)$ that $\hat{f}: \kappa(Z \times X) \rightarrow Y$ is continuous.

For the converse, assume that $\widehat{f}: \kappa(Z \times X) \rightarrow Y$ is continuous. This means that the restrictions of $\hat{f}$ to subspaces $Z \times K_{i}$ are continuous, and Proposition 3 implies that the composites $R_{i} \circ f: Z \rightarrow Y^{K_{i}}$ are continuous. But as $X$ is compactly generated, a function $\phi: X \rightarrow Y$ is continuous if and only if all restrictions $\left.\phi\right|_{K_{i}}: K_{i} \rightarrow Y$ are continuous. This means that the obvious map $Z \rightarrow \prod_{i=1}^{\infty} Y^{K_{i}}$, whose components are $R_{i} \circ f$, maps into the image of the embedding $(*)$ and therefore yields a continuous function $Z \rightarrow Y^{X}$ which is precisely $f$.

Lemma 6. Let $K$ be a compact regular space. The topologies $\kappa((Z \times K) \times X)$ and $\kappa(Z \times X) \times K$ (viewed as topologies on $Z \times X \times K)$ coincide.

We note that this is a corollary of the much more general Theorem 1.15 of Dydak [14]. (Since $K$ is compact regular, it is locally compact according to the definition in [14].) For the sake of completeness, we provide an independent proof (along slightly different lines).

Proof. We show that the two topologies have the same continuous maps into an arbitrary space $Y$. By definition, $f: \kappa(Z \times X \times K) \rightarrow Y$ is continuous if and only if the restrictions $f_{C}: Z \times C \times K \rightarrow Y$ (for compact $C \subset X$ ) are continuous which, by Proposition 3, is if and only if their adjoints $\hat{f}_{C}: Z \times C \rightarrow Y^{K}$ are continuous. The latter is if and only if the map $\widehat{f}: \kappa(Z \times X) \rightarrow Y^{K}$ is continuous and this in turn if and only if $f: \kappa(Z \times X) \times K \rightarrow Y$ is continuous, by another application of Proposition 3. This finishes the proof.

Let $(Z, A)$ be a topological pair (no separation properties assumed). Then $A$ is $P$-embedded in $Z$ if continuous pseudometrics on $A$ extend to continuous pseudometrics on $Z$. Also, $A$ is a zero set in $Z$ if there exists a continuous function $\phi: Z \rightarrow \mathbb{R}$ with $A=\phi^{-1}(0)$. If $A$ is a $P$-embedded zero set, it is called $P_{0}$-embedded.

For example, every closed subset of a metrizable space is $P_{0}$-embedded.

We need $P$-embeddings in the context of Morita's homotopy extension theorem (which in fact characterizes ANR spaces; see Stramaccia [15]).

Theorem 7 (Morita [8]). If $A$ is $P_{0}$-embedded in the topological space $Z$, then the pair $(Z, A)$ has the homotopy extension property with respect to all ANR spaces. That is, if $Y$ is an ANR, if $g: Z \times\{0\} \rightarrow Y$ and $h: A \times[0,1] \rightarrow Y$ are continuous maps that agree pointwise on $A \times\{0\}$, then there exists a continuous map $H: Z \times[0,1] \rightarrow Y$ extending both $g$ and $h$.

Lemma 8. Let $E$ be a Fréchet space and let $X$ be a compactly generated hemicompact (not necessarily Hausdorff) space. Then $E^{X}$ is also a Fréchet space.

Proof. One verifies readily that $E^{X}$ is a topological vector space and that the subbasic open sets $G(K, V)$, where $V$ are convex neighbourhoods of 0 in $E$, constitute a convex local base for $E^{X}$ (see Schaefer [16], page 80). If $E$ is metrizable (by an invariant metric), then $E^{X}$ is metrizable by the (invariant) metric $(* *)$ above. If $E$ is complete, then so is $E^{X}$ since $X$ is compactly generated (see, e.g., Willard [6, Theorem 43.11]).

Proposition 9. Let $A$ be P-embedded in $Z$ and let $X$ be a compactly generated hemicompact space. Then the subset $A \times X$ is $P$-embedded in $\kappa(Z \times X)$.

A result due to Alò and Sennott (see [17, Theorem 1.2]) shows that $A$ is $P$-embedded in $Z$ if and only if every continuous function from $A$ to a Fréchet space extends continuously over $Z$. Proposition 9 seems to be the right way of generalizing the equivalence (1) $\Leftrightarrow$ (2) of Theorem 2.4 in [17].

For a closed subset $A$ of $Z$, the topology $\kappa(A \times X)$ coincides with the topology that the set $A \times X$ inherits from $\kappa(Z \times X)$. For arbitrary $A$, the two topologies may differ, but note that $\kappa(A \times X)$ is always finer than the subspace topology.

Proof. Let $E$ be a Fréchet space and let $f: A \times X \rightarrow E$ be a continuous map where $A \times X$ is understood to inherit its topology from $\kappa(Z \times X)$. Precomposing with the continuous identity $\kappa(A \times X) \rightarrow A \times X$ and using Lemma 5 , we obtain a continuous map $A \rightarrow E^{X}$. By Lemma $8, E^{X}$ is also a Fréchet space and as $A$ is $P$-embedded in $Z$, the function $\widehat{f}$ extends continuously to $\widehat{F}: Z \rightarrow E^{X}$. Reapplying Lemma $5, \widehat{F}$ induces the desired extension $F: \kappa(Z \times X) \rightarrow E$.

Proof of Theorem 1. Let $Z$ be metrizable and let $f: A \rightarrow Y^{X}$ be a continuous map defined on the closed subset $A$ of $Z$. By assumption, $Y^{X}$ has the homotopy type of an ANR; hence $f$ admits a neighbourhood extension up to homotopy. That is, there exist a continuous map $g: U \rightarrow Y^{X}$ where $U$ is open and contains $A$ and a homotopy $h: A \times[0,1] \rightarrow Y^{X}$ beginning in $\left.g\right|_{A}$ and ending in $f$. 
Let $h \sqcup g: A \times[0,1] \cup U \times\{0\} \rightarrow Y^{X}$ denote the continuous union of the two, with adjoint $\widehat{h} \sqcup \hat{g}: \kappa((A \times[0,1] \cup U \times$ $\{0\}) \times X) \rightarrow Y$. As $A \times[0,1] \cup U \times\{0\}$ is closed in $A \times U$, the map $\widehat{h} \sqcup \hat{g}$ is continuous with respect to the topology that $(A \times[0,1] \cup U \times\{0\}) \times X$ inherits from $\kappa(U \times[0,1] \times X)$. Under the homeomorphism $\kappa(U \times[0,1] \times X) \approx \kappa(U \times X) \times[0,1]$ of Lemma 6, the map $\widehat{h} \sqcup \hat{g}$ corresponds to a continuous map $\widehat{k}: \kappa(A \times X) \times[0,1] \cup \kappa(U \times X) \times\{0\} \rightarrow Y$.

Obviously, as $A$ is a zero set in $U$, the product $A \times X$ is a zero set in $U \times X$ with respect to the Cartesian product topology. A fortiori, $A \times X$ is a zero set in $\kappa(U \times X)$. Hence, by Proposition 9, the set $A \times X$ is $P_{0}$-embedded in $\kappa(U \times X)$. Theorem 7 yields an extension of $\widehat{k}$ to $K: \kappa(U \times$ $X) \times[0,1] \rightarrow Y$. Reapplying Lemma 6 and Lemma 5, $K$ induces a continuous function $k: U \times[0,1] \rightarrow Y^{X}$. Level 1 of this homotopy is a continuous extension of $f$ over the neighbourhood $U$. Therefore, $Y^{X}$ is an ANR.

Corollary 10. If $C$ is a compact space and $Y$ is an ANR, then $Y^{C}$ is an ANR.

Proof. By Theorem 3 of Milnor [3], $Y^{C}$ has CW homotopy type.

Corollary 10 was proved independently by Yamashita [18] (with the additional requirement that $C$ be Hausdorff) but the author of this note has not seen it elsewhere for nonmetrizable compacta $C$. From the point of view of $P$ embeddings, however, Corollary 10 encodes a long-known fact (see Przymusiński [19, Theorem 3]): if $A$ is $P$-embedded in $Z$ and $X$ is a compact space, then $A \times X$ is $P$-embedded in $Z \times X$.

Proof of Corollary 2. Suppose that $Y^{X}$ is metrizable. If $Y$ contains an arc (which is if and only if it has a nontrivial path component), it follows that $[0,1]^{X}$ is metrizable. Since $X$ is a Tychonoff space, points in $X$ can be separated from compact sets in $X$ by means of continuous functions $X \rightarrow$ $[0,1]$. The proof of Theorem 8 of Arens [5] can be adapted almost verbatim to render $X$ hemicompact. The statement of Corollary 2 follows immediately from Theorem 1 .

\section{Appendix}

\section{A Characterization of Compactly Generated Hemicompact Spaces}

A function $q: Z \rightarrow X$ will be called weakly proper if for each compact subset $K$ of $X$ there exists a compact subset $L$ of $Z$ so that $q(L)=K$. Note that a weakly proper map is necessarily surjective. Finally, recall that $Z$ is a $\sigma$-compact space if $Z$ is the union of a countable collection of its compact subsets.

Proposition A.1. The topological space $X$ is compactly generated and hemicompact if and only if there exists a $\sigma$-compact locally compact space $Z$ with a weakly proper quotient map $q: Z \rightarrow X$.
Proof. Let $X$ be a compactly generated hemicompact space with its sequence of distinguished compact subsets $\left\{K_{i}\right\}$ and let $q$ denote the obvious surjective map from the disjoint union $\bigsqcup_{i} K_{i}=: Z$ to $X$. Clearly, a set $A$ in $X$ is closed if and only if $A \cap K_{i}$ is closed in $K_{i}$ for all $i$. Thus, $q$ is a quotient map. Tautologically, $Z$ is both $\sigma$-compact and locally compact, and hemicompactness renders $q$ weakly proper.

For the reverse implication, suppose first that $Z$ is a compactly generated hemicompact space with distinguished compact subsets $\left\{L_{i}\right\}$ and $q: Z \rightarrow X$ is a weakly proper quotient map. Clearly, as $q$ is weakly proper, $X$ is hemicompact with distinguished compact subsets $\left\{q\left(L_{i}\right)\right\}$. To see that $X$ is compactly generated, let $f: X \rightarrow W$ be a function that is continuous on all compact sets. Hence, if $L$ is a compact subset of $Z, f \circ q$ is continuous on the saturation $q^{-1}(q(L))$. Consequently, $f$ is continuous on $L$. Since $Z$ is compactly generated, $f \circ q$ is continuous and since $q$ is a quotient map, so also is $f$. Since this holds for all $f, X$ is compactly generated.

Finally, suppose that $Z$ is a $\sigma$-compact locally compact space. Then $Z$ is compactly generated. (See, e.g., [6], 43.9.) Since $Z$ is locally compact, it has an open cover $\mathcal{U}$ consisting of relatively compact sets. As $Z$ is the union of countably many compact sets, $\mathscr{U}$ has a countable subcover $\left\{U_{j} \mid j=\right.$ $1,2,3, \ldots\}$. The sequence of compact sets $L_{i}=\cup_{j=1}^{i} \bar{U}_{j}, i=$ $1,2,3, \ldots$, exhibits $Z$ as hemicompact. This completes the proof.

\section{Acknowledgments}

The author is grateful to Atsushi Yamashita for posing the question that has led to the results presented here and to the referee for recommending that a characterization of compactly generated hemicompact spaces in the sense of Proposition A.1 be included in the paper. The author was supported in part by the Slovenian Research Agency Grants P1-0292-0101 and J1-4144-0101.

\section{References}

[1] S. Mardešić, "Absolute neighborhood retracts and shape theory," in History of Topology, I. M. James, Ed., chapter 9, pp. 241-269, North-Holland, Amsterdam, Netherlands, 1999.

[2] K. Kuratowski, "Sur les espaces localement connexes et peaniens en dimensions n," Fundamenta Mathematicae, vol. 24, no. 1, pp. 269-287, 1935.

[3] J. Milnor, "On spaces having the homotopy type of a CWcomplex," Transactions of the American Mathematical Society, vol. 90, no. 2, pp. 272-280, 1959.

[4] R. Cauty, "Une caractérisation des rétractes absolus de voisinage," Fundamenta Mathematicae, vol. 144, no. 1, pp. 11-22, 1994.

[5] R. F. Arens, "A topology for spaces of transformations," Annals of Mathematics, vol. 47, no. 3, pp. 480-495, 1946.

[6] S. Willard, General Topology, Addison-Wesley, Reading, Mass, USA, 1970 .

[7] J. Smrekar and A. Yamashita, "Function spaces of CW homotopy type are Hilbert manifolds," Proceedings of the American Mathematical Society, vol. 137, no. 2, pp. 751-759, 2009. 
[8] K. Morita, "On generalizations of Borsuk's homotopy extension theorem," Fundamenta Mathematicae, vol. 88, no. 1, pp. 1-6, 1975.

[9] J. Smrekar, "Compact open topology and CW homotopy type," Topology and Its Applications, vol. 130, no. 3, pp. 291-304, 2003.

[10] J. Smrekar, "Homotopy type of mapping spaces and existence of geometric exponents," Forum Mathematicum, vol. 22, no. 3, pp. 433-456, 2010.

[11] S.-t. Hu, Theory of Retracts, Wayne State University Press, Detroit, Mich, USA, 1965.

[12] R. Cauty, "Sur les espaces d'applications dans les CWcomplexes," Archiv der Mathematik, vol. 27, no. 3, pp. 306-311, 1976.

[13] I. M. James, General Topology and Homotopy Theory, Springer, New York, NY, USA, 1984.

[14] J. Dydak, "Covariant and contravariant points of view in topology with applications to function spaces," Topology and Its Applications, vol. 94, no. 1-3, pp. 87-125, 1999.

[15] L. Stramaccia, "P-embeddings, AR and ANR spaces," Homology, Homotopy and Applications, vol. 5, no. 1, pp. 213-218, 2003.

[16] H. H. Schaefer, Topological Vector Spaces, vol. 3 of Graduate Texts in Mathematics, Springer, New York, NY, USA, 1971.

[17] R. A. Alò and L. Sennott, "Collectionwise normality and the extension of functions on product spaces," Fundamenta Mathematicae, vol. 76, no. 3, pp. 231-243, 1972.

[18] A. Yamashita, Private Communication.

[19] T. Przymusiński, "Collectionwise normality and extensions of continuous functions," Fundamenta Mathematicae, vol. 98, no. 1, pp. 75-81, 1978. 


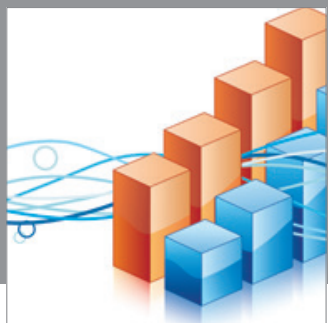

Advances in

Operations Research

mansans

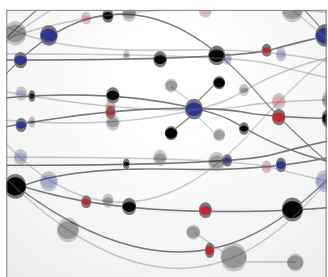

The Scientific World Journal
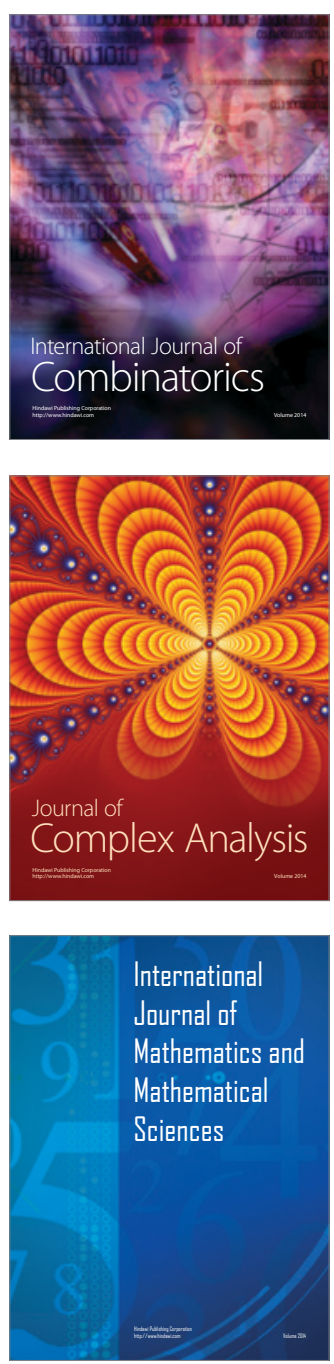
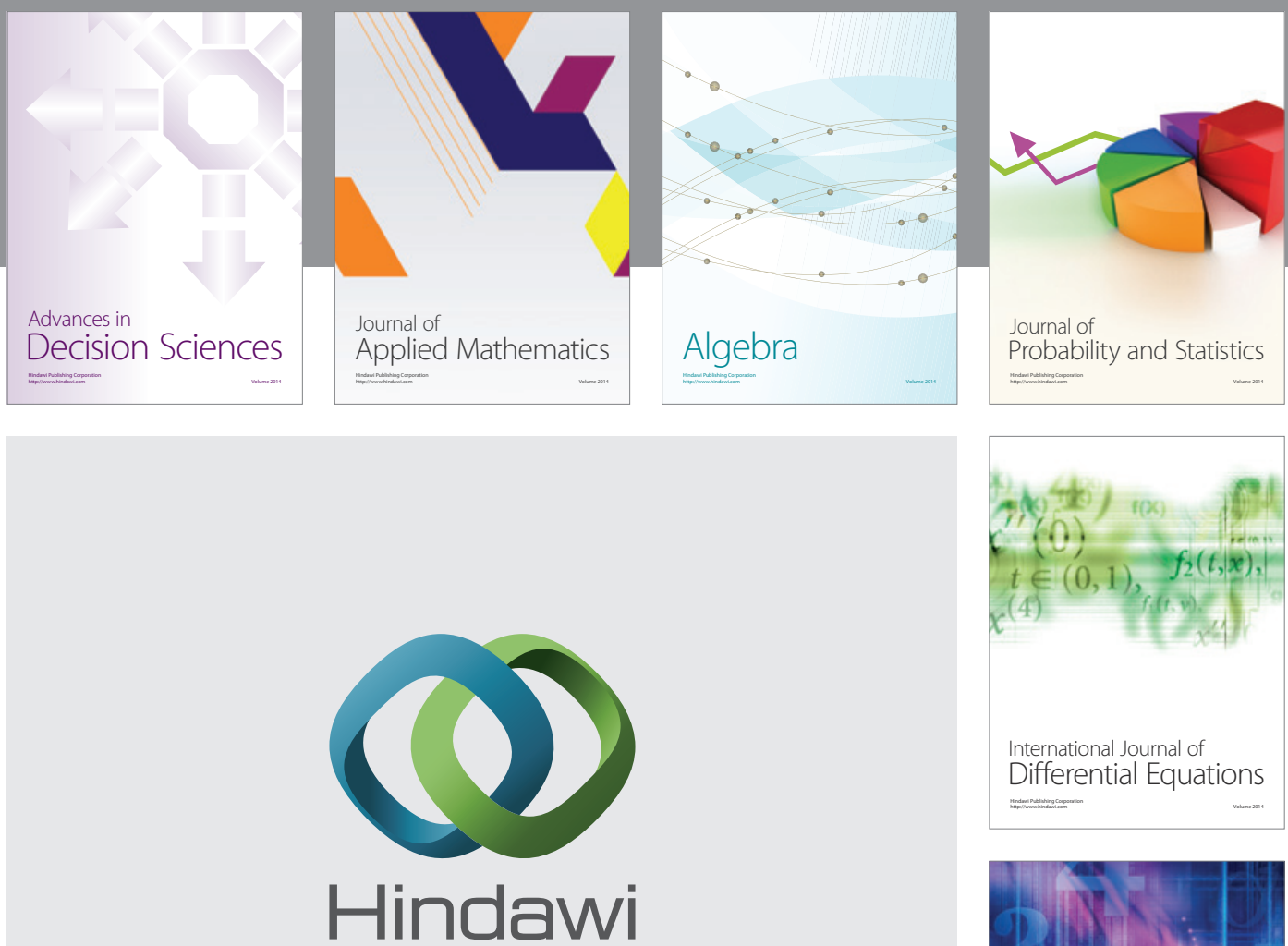

Submit your manuscripts at http://www.hindawi.com
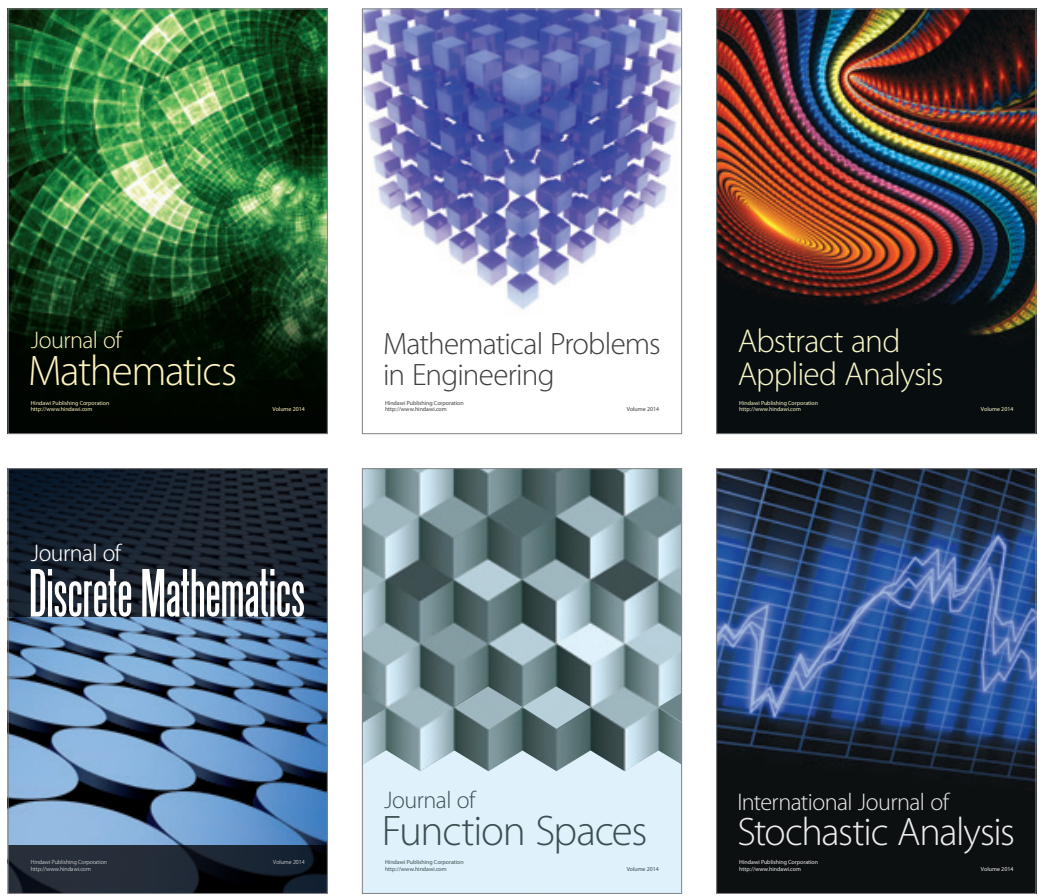

Journal of

Function Spaces

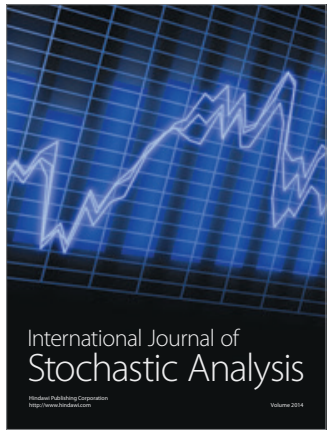

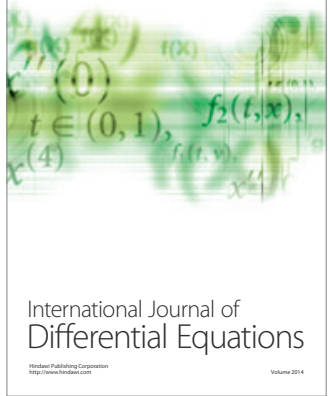
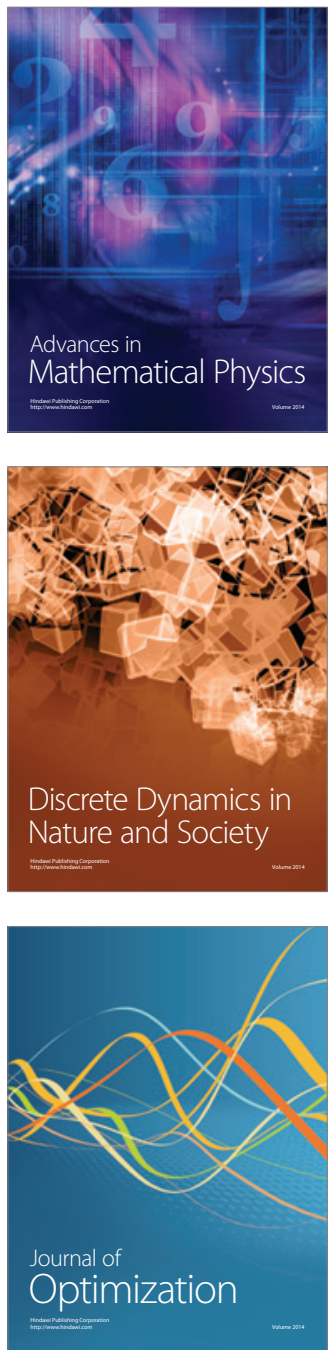